\title{
Optimization of the Epitaxial Growth of Undoped GaN Waveguides in GaN-Based Laser Diodes Evaluated by Photoluminescence
}

\author{
C. NETZEL $\odot,{ }^{1,2}$ V. HOFFMANN,${ }^{1}$ S. EINFELDT,${ }^{1}$ and M. WEYERS ${ }^{1}$ \\ 1.-Ferdinand-Braun-Institut, Leibniz-Institut für Höchstfrequenztechnik, Gustav-Kirchhoff- \\ Straße 4, 12489 Berlin, Germany. 2.—e-mail: Carsten.Netzel@fbh-berlin.de
}

\begin{abstract}
Non-intentionally doped c-plane GaN layers are generally employed as p-side waveguide layers in violet/blue-emitting laser diodes. The recombination and diffusion of charge carriers in the p-side $\mathrm{GaN}$ waveguide influence the injection efficiency of holes into the InGaN quantum wells of these devices. In this study, the non-radiative recombination and the diffusivity in the [000-1] direction for charge carriers in such GaN layers are investigated by the photoluminescence of buried InGaN quantum wells, in addition to the GaN photoluminescence. The vertical charge carrier diffusion length and the diffusion constant in GaN were determined by evaluating the intensity from InGaN quantum wells in different depths below a top GaN layer. Additionally, the intensity from the buried InGaN quantum wells was found to be more sensitive to variations in the non-radiative recombination rate in the GaN layer than the intensity from the GaN itself. The study enables conclusions to be drawn on how the growth of a p-side $\mathrm{GaN}$ waveguide layer has to be optimized: (1) The charge carrier diffusivity in the [000-1] direction at device operation temperature is limited by phonon scattering and can be only slightly improved by material quality. (2) The use of TMGa (trimethylgallium) instead of TEGa (triethylgallium) as a precursor for the growth of GaN lowers the background silicon doping level and is advantageous for a large hole diffusion length. (3) Small growth rates below $0.5 \mu \mathrm{m} / \mathrm{h}$ when using TMGa or below $0.12 \mu \mathrm{m} / \mathrm{h}$ when using TEGa enhance non-radiative recombination. (4) A V/III gas ratio of 2200 or more is needed for low non-radiative recombination rates in GaN.
\end{abstract}

Key words: Gallium nitride, MOVPE growth, charge carrier diffusion, photoluminescence

\section{INTRODUCTION}

GaN/InGaN-based violet/blue-emitting LEDs and laser diodes have achieved high emission power and high quantum efficiency. ${ }^{1,2}$ Nevertheless, the IIInitride semiconductor layers used for these devices are far from being crystallographically perfect. High densities of dislocations $\left(\sim 10^{6}-10^{9} \mathrm{~cm}^{-2}\right)$ as well as

(Received February 17, 2020; accepted April 29, 2020; published online May 14, 2020) intrinsic and extrinsic point defects $\left(\sim 10^{15}\right.$ $10^{17} \mathrm{~cm}^{-3}$ ) result from heteroepitaxial growth which is associated with thermal and lattice mismatch, differences in the surface desorption temperature of indium, gallium, and aluminum, the use and insufficient cracking of metalorganic precursors, and contamination from the growth environment. The limited crystal perfection complicates the precise determination of fundamental material parameters, e.g. radiative recombination times, polarization coefficients, or lattice constants. On 
the other hand, it allows for extensive material and device optimization.

High material purity and low dislocation density are required for undoped $\mathrm{GaN}$ layers which are generally used as p-side waveguides in InGaN/GaNbased laser diodes, to avoid strong absorption of the laser mode by non-ionized acceptors or free carrier absorption in p-type layers doped with $\mathrm{Mg} .{ }^{3}$ High hole diffusivity and a low probability of non-radiative recombination, both expected for material of high crystal perfection, would be beneficial for this layer, as it would ensure efficient carrier injection into the quantum wells. The efficiency of an electron blocking layer can thus be supported. The characterization of a non-intentionally doped (nid) GaN waveguide layer with respect to its electrical properties is generally limited to measuring the electron density and mobility. Optimization of the nid GaN waveguide layer based on the laser performance is a lengthy process. Methods like secondary ion mass spectrometry (SIMS) to determine the density of foreign atoms or positron annihilation spectroscopy to measure vacancies are complex and usually limited to concentrations in the range above $10^{15}$ $10^{17} \mathrm{~cm}^{-3}$ for the most relevant point defects in $\mathrm{GaN}^{4,5}$

In this study, we use photoluminescence (PL) spectroscopy as a characterization method. PL is applied to test samples which are largely identical to a laser diode heterostructure. A GaN layer on top of the InGaN quantum wells, which would be the pside waveguide in a laser structure, is grown with different thicknesses and under different growth conditions. The experiment provides information (i) on the non-radiative recombination in the top $\mathrm{GaN}$ layer via the PL intensity from the $\mathrm{GaN}$, and (ii) on the charge carrier diffusivity in the top GaN layer via the PL intensity from the InGaN quantum wells.

\section{EXPERIMENTAL DETAILS}

All samples were grown by metalorganic vaporphase epitaxy (MOVPE) on 2-inch sapphire templates in an Aixtron AIX 200/4-RF-S system with standard precursors triethylgallium (TEGa) or trimethylgallium (TMGa), trimethylindium, triethylaluminum, disilane, and ammonia. The complete heterostructures contain a buffer, an n-side, and quantum wells, similar to a GaN/InGaN-based laser diode (compare Fig. 1). This ensures reactor conditions and a strain situation for the following growth of the top GaN layer which are similar to those used for the p-side GaN waveguide of a laser diode. In detail, the heterostructure includes a thin GaN nucleation layer, $2.5 \mu \mathrm{m}$ nid $\mathrm{GaN}$ as bottom buffer layer, $2.5 \mu \mathrm{m}$ GaN:Si as n-doped top buffer layer, an $\mathrm{Al}_{0.12} \mathrm{Ga}_{0.88} \mathrm{~N} / \mathrm{GaN}: \mathrm{Si}$ superlattice as $\mathrm{n}$ side cladding, $150 \mathrm{~nm}$ GaN:Si as n-side waveguide ([Si] $\sim 5 \times 10^{17} \mathrm{~cm}^{-3}$ ), and three nominally $3.5 \mathrm{~nm}$ thick $\mathrm{In}_{0.1} \mathrm{Ga}_{0.9} \mathrm{~N}$ quantum wells separated by
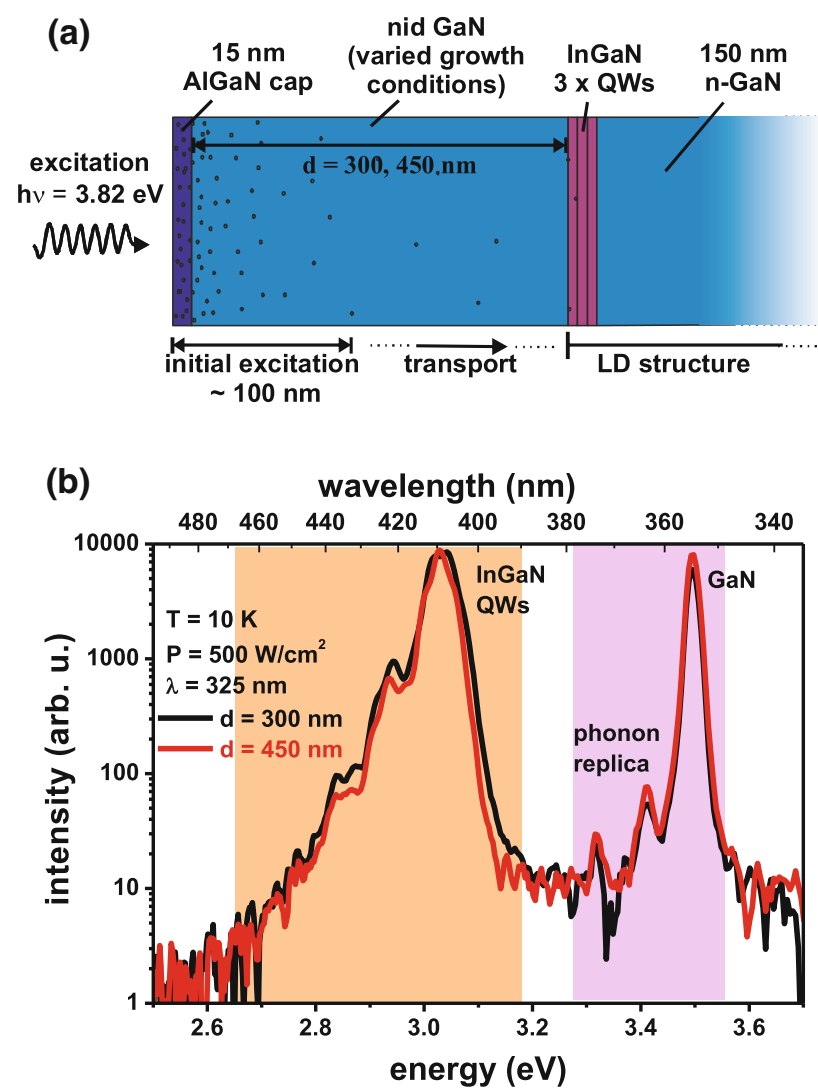

Fig. 1. (a) Sketch of the investigated heterostructures. The growth conditions of the nid GaN layer were varied. (b) Exemplary PL spectra from heterostructures with different top GaN layer thicknesses, $d$, showing the emission from the InGaN quantum wells and the GaN top layer. The energy ranges for integrating the intensity are highlighted.

$11 \mathrm{~nm}$-thick $\mathrm{In}_{0.02} \mathrm{Ga}_{0.98} \mathrm{~N}$ barriers. The quantum well emission wavelength was around $410 \mathrm{~nm}$ at room temperature. The investigated $\mathrm{GaN}$ layer was grown on top of the quantum wells, without intentional doping, using a reactor pressure and temperature of $200 \mathrm{mbar}$ and $1050^{\circ} \mathrm{C}$, respectively. The GaN layer thickness, V/III ratio, growth rate, and the gallium precursor were varied for this layer. The GaN layer was capped by a $15 \mathrm{~nm}$-thick $\mathrm{Al}_{0.15} \mathrm{Ga}_{0.85} \mathrm{~N}$ layer to exclude effects from varying surface conditions on the PL intensity that can appear during extended or repeated PL experiments. ${ }^{6}$ Exemplary PL spectra are shown in Fig. 1b. The InGaN peak energy was kept constant. The PL intensity was determined by spectral integration over the main peak and associated phonon replicas, as illustrated by the marked energy ranges in Fig. $1 b$.

A $325 \mathrm{~nm}$ continuous-wave $\mathrm{HeCd}$ laser with maximum excitation power density of $500 \mathrm{~W} / \mathrm{cm}^{2}$ on a spot $70 \mu \mathrm{m}$ in diameter was used for $\mathrm{PL}$ excitation. Temperature-dependent measurements were performed between $10 \mathrm{~K}$ and $310 \mathrm{~K}$ using a closed-cycle helium cryostat. 


\section{RESULTS AND DISCUSSION}

Diffusion lengths, $L_{\text {diff, }}$ were determined by evaluating the PL intensity from InGaN quantum wells buried under GaN layers of different thicknesses. This approach makes several demands on the analyzed heterostructures, the recombination processes, and the PL experiment which have been discussed in previous publications. ${ }^{7,8}$ Under suitable conditions, the PL intensities from InGaN quantum wells in different depths, $d$ and $d+\Delta d$, allow for the determination of $L_{\text {diff }}$ according to Eq. 1:

$$
I(d+\Delta d)=I(d) \cdot \exp \left(-\frac{\Delta d}{L_{\text {diff }}}\right)
$$

Diffusion constants, $D$, can be derived from the diffusion lengths by Eq. 2 if the effective recombination times, $\tau_{\mathrm{GaN}}$, have been previously determined for GaN bulk layers grown under similar conditions.

$$
D=\frac{L_{\text {diff }}^{2}}{\tau_{\mathrm{GaN}}}
$$

Temperature-dependent diffusion lengths and diffusion constants in the [000-1] direction for nid GaN layers $(\mathrm{V} / \mathrm{III}$ ratio $=1100, \mathrm{TMGa}$ usage, growth rate $=1 \mu \mathrm{m} / \mathrm{h}$, thickness $=300 \mathrm{~nm}$ and $450 \mathrm{~nm})$ on sapphire substrates are presented in Fig. 2. A more detailed description of the determination of these values, underlying PL intensity data, and error ranges can be found elsewhere. ${ }^{7}$ For temperatures beyond $170 \mathrm{~K}$, the diffusivity of charge carriers decreases. The temperature dependence in this range lies between the temperature dependencies theoretically expected from scattering by acoustic $\left(\propto T^{-0.5}\right)$ or optical phonons $\left(\propto T \times \exp \left(E_{\mathrm{LO}} /\left(k_{\mathrm{B}}\right.\right.\right.$ $T$ )). ${ }^{9,10}$ Here, $E_{\mathrm{LO}}$ is the phonon energy, and $k_{\mathrm{B}}$ the Boltzmann constant. Impurity scattering would increase the diffusion constant when increasing the

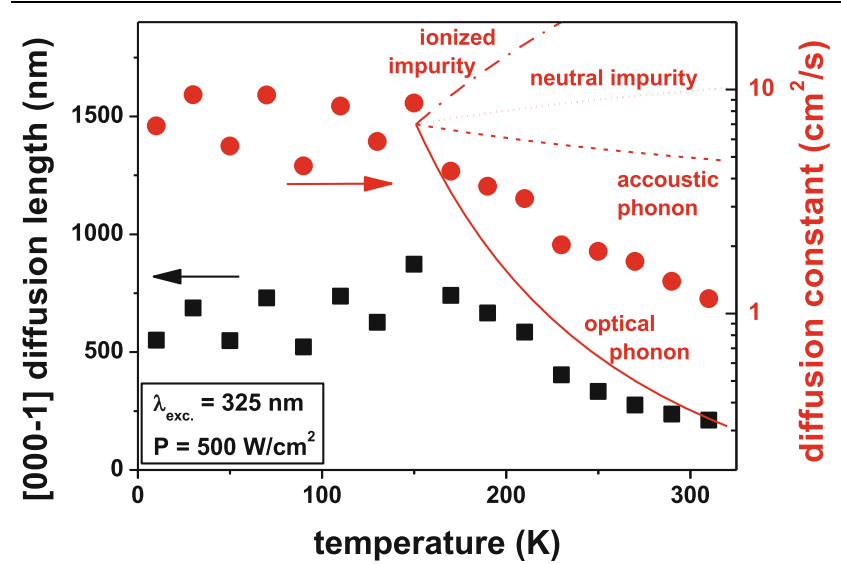

Fig. 2. Temperature-dependent diffusion lengths and diffusion constants in the [000-1] direction for charge carriers in the top GaN layer. Additionally, theoretical temperature dependencies for the diffusion constant are shown, based on phonon and impurity scattering and normalized to $7 \mathrm{~cm}^{2} / \mathrm{s}$ at $150 \mathrm{~K}$. temperature, both for ionized impurities $\left(\propto T^{2.5}\right)$ and neutral impurities $\left(\propto T^{0.5}\right){ }^{9,11}$ Accordingly, the diffusion constant in the [000-1] direction for $\mathrm{GaN}$ at room temperature or beyond is based on the interaction with phonons. Therefore, it depends mainly on the density of phonons and the sample temperature, and only slightly on the material quality, e.g. point defects. The MOVPE growth conditions of the GaN may thus change the point defect density, but should have little effect on the charge carrier diffusivity in devices. Nevertheless, the diffusion length is related to the GaN purity, since it depends on the effective recombination time and therefore also on the non-radiative recombination rate in the material.

Figure 3 shows the temperature dependence of the GaN and InGaN PL intensities for a set of heterostructures where the V/III ratio during MOVPE growth of the $450 \mathrm{~nm}$-thick top GaN layer was varied between 200 and 2200 (gallium precursor $=\mathrm{TMGa}$, growth rate $=1 \mu \mathrm{m} / \mathrm{h}$ ). Regardless of the temperature, the PL intensities increase with the V/III ratio. For a V/III ratio that is higher than 1000 , the PL intensities seem to saturate.
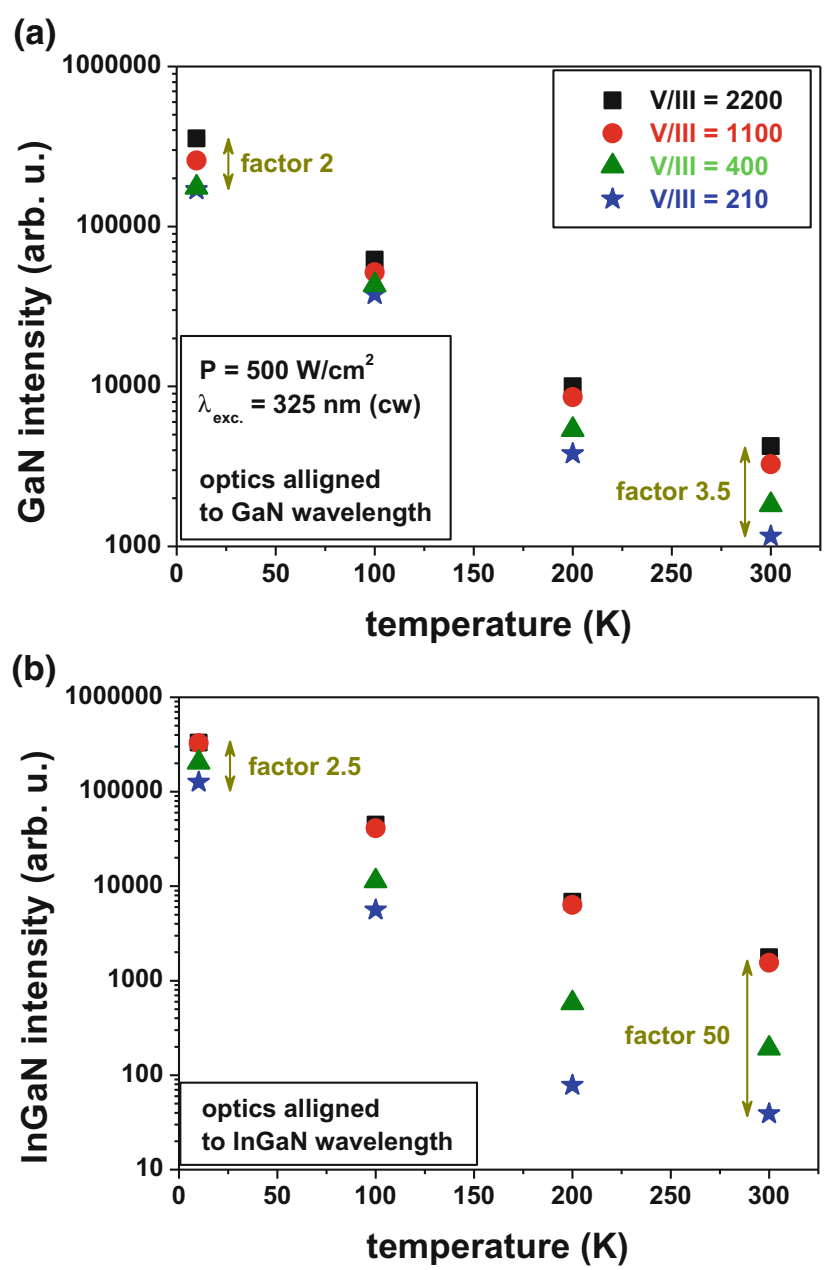

Fig. 3. Temperature-dependent $\mathrm{GaN}$ (a) and InGaN (b) $\mathrm{PL}$ intensities for heterostructures with a $450 \mathrm{~nm}$-thick top GaN layer grown with different $\mathrm{V} / \mathrm{III}$ ratios. 
Remarkably, the variation of the PL intensity with the V/III ratio is much stronger for the InGaN quantum well emission (Fig. 3b) than for the GaN emission (Fig. 3a), particularly around room temperature. This effect results from the small diffusion length in the top GaN layer at room temperature. While the GaN PL intensity at room temperature is mainly defined by the ratio of radiative and nonradiative recombination rates in the top GaN layer, the InGaN quantum well PL intensity is determined by a similar ratio for the InGaN quantum wells but multiplied by a factor that describes how many charge carriers reach the quantum wells, i.e. that have not recombined in the top GaN layer. Therefore, the InGaN quantum well PL intensity decreases exponentially with decreasing diffusion length or decreasing effective recombination time in the top GaN layer. Accordingly, the InGaN quantum well PL intensity at room temperature is more sensitive to non-radiative recombination in the $\mathrm{GaN}$ either when the top GaN layer is thick or when the non-radiative recombination in the $\mathrm{GaN}$ is strong and reduces the diffusion length significantly. The latter is the case when the V/III ratio is below 400 , as can be seen in Fig. 3 for the data at room temperature (factor 50 for InGaN instead of factor 3.5 for GaN). The high sensitivity of the InGaN emission intensity is advantageous for sensing small differences in the GaN layer quality. Concerning the optimization of the GaN layer and the desired limitation of non-radiative recombination, the PL data in Fig. 3 suggests a high V/III ratio of $\geq 2200$ for maximum crystal perfection during MOVPE growth in the reactor system used here. The result is coherent with previous observations for MOVPE-grown GaN bulk layers. ${ }^{12}$ It is assumed that the enhanced non-radiative recombination for a low V/III ratio results from an increased density of vacancies in the crystal. ${ }^{13} \mathrm{~A}$ higher carbon concentration in the case of a lower V/III ratio can also be expected. ${ }^{14}$ Additional carbon would cause stronger non-radiative recombination. ${ }^{15}$ However, meaningful studies on this topic, which may define the main cause for low internal quantum efficiency in GaN layers, are still missing.

Figure 4 shows room-temperature GaN PL intensities for a set of heterostructures fabricated with different gallium precursors (TEGa or TMGa) and growth rates. The top GaN layer thickness was kept constant at $450 \mathrm{~nm}$. The values are plotted against the intensity ratio of the donor-bound exciton transition $\left(\mathrm{D}^{\circ} \mathrm{X}\right)$ and the free exciton transition (FX) from low-temperature PL measurements carried out with high spectral resolution. Examples of such spectra are presented in Fig. 5. The intensity ratio of the two transitions is known to increase with increasing donor concentration in the material. ${ }^{16}$ The GaN PL intensity at room temperature is stronger when TEGa was used instead of TMGa (Fig. 4). However, the use of TEGa comes with an enhanced doping with donors and, consequently,

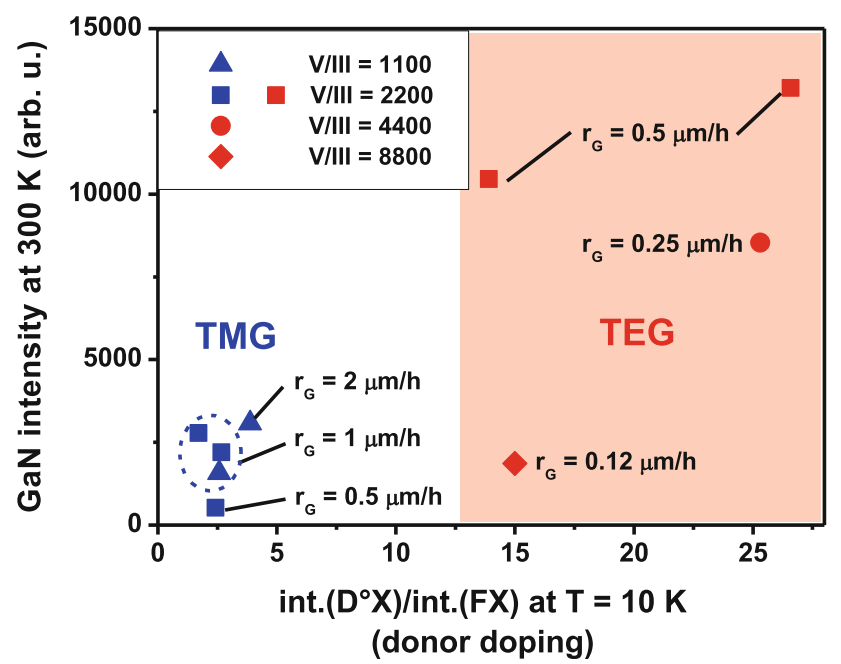

Fig. 4. GaN PL intensity at room temperature plotted against the intensity ratio of donor-bound excitons to free excitons at $10 \mathrm{~K}$. The latter is a figure of merit for the donor doping level. The precursor (TMGa or TEGa) used, growth rate $r_{G}$, and V/III ratio are marked.

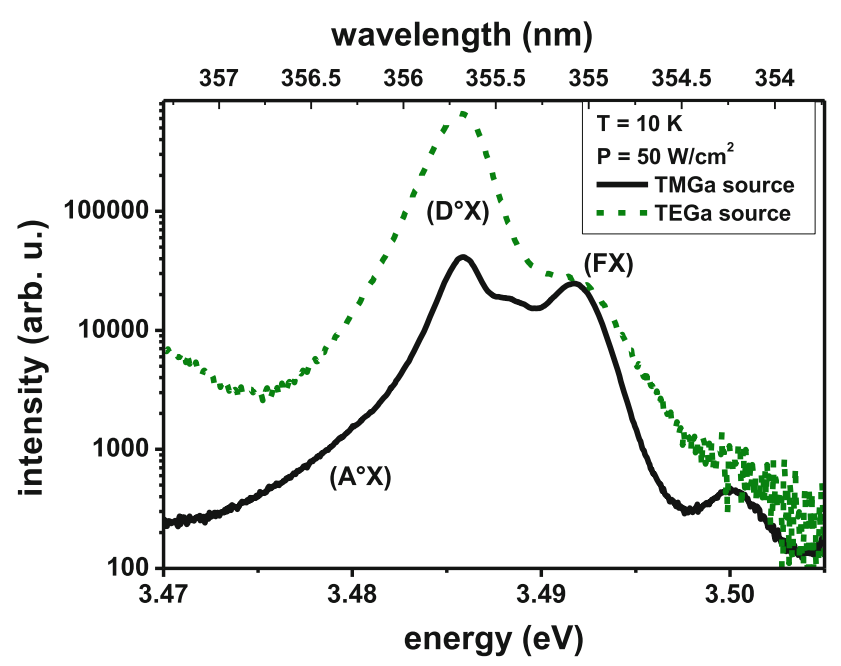

Fig. 5. Low-temperature, high-resolution PL spectra as examples of the GaN emission in the case of using TMGa or TEGa as precursor.

higher free carrier density at room temperature. In this case, the high PL intensity does not indicate a low non-radiative recombination rate and high purity of the GaN, but contamination of the material with donors. The InGaN quantum well PL intensity is then no longer a good figure of merit for the quality of the top GaN layer, because the high carrier density results in efficient radiative recombination in the GaN which limits the diffusion length. Results from SIMS, shown in Fig. 6, confirm the expectations with respect to the donor concentration. The silicon concentration in the top GaN layer increased from $10^{16} \mathrm{~cm}^{-3}$ to more than $10^{17} \mathrm{~cm}^{-3}$ when it was grown with TEGa instead of TMGa. Similar effects have been observed by other groups in the past. ${ }^{17,18}$ This effect very likely results from the lower purity of the TEGa source compared with the TMGa source. This is supported 


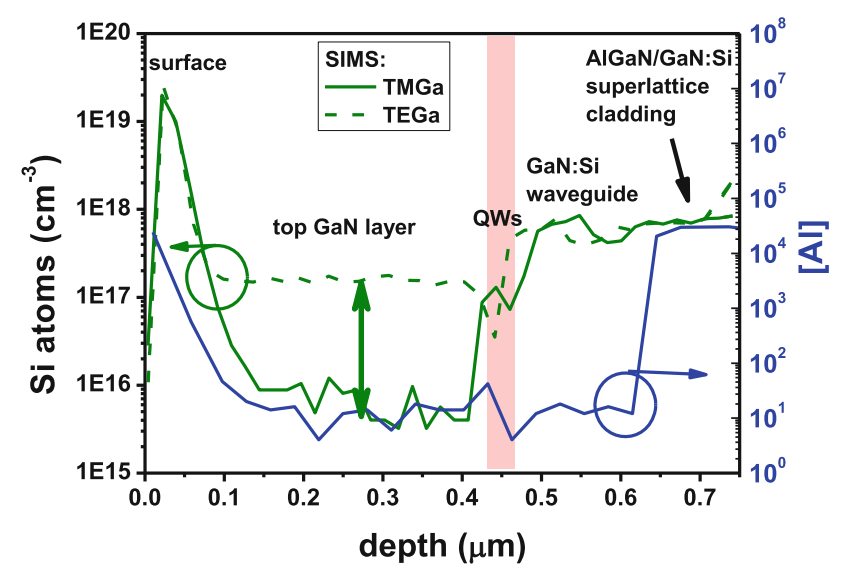

Fig. 6. Depth profile of the silicon concentration and aluminum concentration in the heterostructures when either TMGa or TEGa were used as precursors for the growth of the top GaN layer as determined by SIMS. The step in the aluminum signal indicates the interface between the cladding layer and the $n$-side waveguide. The position of the InGaN quantum wells is marked. Increased concentrations for depths below $100 \mathrm{~nm}$ are measurement artefacts caused by surface contamination.

by information on the sources' purity provided by the producer. It remains an open question whether differences in the growth process, e.g. caused by the varying binding strength of the organic components, may also contribute. For the optimization of a p-side GaN waveguide, enhanced silicon doping is in any case disadvantageous, because it increases the recombination probability for holes which diffuse through this layer toward the InGaN quantum wells. In conclusion, the p-side $\mathrm{GaN}$ waveguide layer should be grown with TMGa as precursor.

The growth rate for the top GaN layer was varied with or without simultaneous changes in the V/III ratio. The growth rates and V/III ratios are given in Fig. 4. Regardless of the precursor used, the GaN PL intensity at room temperature decreases with decreasing growth rate. High V/III ratios of 2200 and above cannot compensate for this drop in intensity. The origin of the enhanced non-radiative recombination for low growth rates is not yet clear. Contamination with extrinsic defects like carbon and a related non-radiative recombination should decrease when the growth rate is reduced. ${ }^{14,15,19}$ One would also assume that adatoms have a higher chance of filling vacancies near the growth front when the growth rate is low. Although there is no explanation for the experimental finding, it can be concluded that the growth rate for a p-side GaN waveguide layer should be at least $1 \mu \mathrm{m} / \mathrm{h}$ under TMGa usage to minimize non-radiative recombination.

\section{CONCLUSION}

PL emission intensities from GaN layers and buried InGaN quantum wells have been analyzed to find optimal MOVPE growth conditions for the undoped $p$-side GaN waveguide of a laser heterostructure which result in large charge carrier diffusivity and minimum non-radiative recombination. The diffusion constant in the [000-1] direction for charge carriers at room temperature was found to be limited by phonon scattering. It is concluded that the hole diffusion constant cannot be improved by optimizing the GaN growth conditions. Using the TMGa precursor instead of TEGa results in a lower silicon background doping level, which is recommended to minimize recombination in a $p$-side waveguide layer. Furthermore, a high V/III ratio of 2200 or more and a high growth rate of at least $1 \mu \mathrm{m} / \mathrm{h}$ using TMGa were found to be advantageous in respect to the reduction of non-radiative recombination in a nid GaN layer.

\section{ACKNOWLEDGMENTS}

Open Access funding provided by Projekt DEAL. This work was supported in part by the European Fund for Regional Development of the European Union in the framework of the Berlin-Polish joint project "From UV to blue - reliable laser sources for environmental monitoring (RelyLa)", administered by the Investitionsbank Berlin within the "Program to promote research, innovation and technologies" (ProFIT) under Contract 10164535 and in part by the German Research Foundation in the Collaborative Research Centre 787.

\section{OPEN ACCESS}

This article is licensed under a Creative Commons Attribution 4.0 International License, which permits use, sharing, adaptation, distribution and reproduction in any medium or format, as long as you give appropriate credit to the original author(s) and the source, provide a link to the Creative Commons licence, and indicate if changes were made. The images or other third party material in this article are included in the article's Creative Commons licence, unless indicated otherwise in a credit line to the material. If material is not included in the article's Creative Commons licence and your intended use is not permitted by statutory regulation or exceeds the permitted use, you will need to obtain permission directly from the copyright holder. To view a copy of this licence, visit http://creativecom mons.org/licenses/by/4.0/.

\section{REFERENCES}

1. S. Masui, Y. Nakatsu, D. Kasahara, and S. Nagahama, Proceedings of SPIE OPTO (2017), p. 101041H-7.

2. D. Feezell and S. Nakamura, C. R. Phys. 19, 113 (2018).

3. D. Feezell and S. Nakamura, Semiconductor Lasers Fundamentals and Applications, ed. A. Baranov and E. Tournié (Cambridge: Woodhead Publishing Limited, 2013), p. Chapter 6.

4. A. Uedono, S. Ishibashi, T. Ohdaira, and R. Suzuki, J. Cryst. Growth 311, 3075 (2009).

5. M.A. Reshchikov, Semiconductors and Semimetals 91: Defects Semiconductors, ed. L. Romano, V. Provitera, and C. Jagadish (Burlington: Academic Press, 2015), pp. 315-367. 
6. C. Netzel, J. Jeschke, F. Brunner, A. Knauer, and M. Weyers, J. Appl. Phys. 120, 095307 (2016).

7. C. Netzel, V. Hoffmann, J.W. Tomm, F. Mahler, S. Einfeldt, and M. Weyers, Phys. Status Solidi B (2020). https://doi.org/ 10.1002/pssb.202000016.

8. J.Y. Duboz, F. Binet, D. Dolfi, N. Laurent, F. Scholz, J. Off, A. Sohmer, O. Briot, and B. Gil, Mater. Sci. Eng. B50, 289 (1997).

9. K. Seeger, Semiconductor Physics (Wien: Springer, 1973), pp. $164-230$

10. N.N. Zinov'ev, L.P. Ivanov, I.G. Lang, S.T. Pavlov, A.V. Prokaznikov, and I.D. Yaroshetskiĩ, Sov. Phys. JETP 57, 1254 (1983).

11. J. Singh, Electronic and Optoelectronic Properties of Semiconductor Structures (Cambridge: Cambridge University Press, 2003), pp. 179-259.

12. O. Briot, J.P. Alexis, M. Tchounkeu, and R.L. Aulombard, Mater. Sci. Eng. B 43, 147 (1997).

13. O. Briot, Group III Nitride Semiconductor Compounds, Physics and Applications, ed. B. Gil (Oxford: Oxford University Press, 1998), pp. 70-122.
14. T. Ciarkowski, N. Allen, E. Carlson, R. McCarthy, C. Youtsey, J. Wang, P. Fay, J. Xie, and L. Guido, Materials 12, 2455 (2019).

15. K. Kojima, F. Horikiri, Y. Narita, T. Yoshida, H. Fujikura, and S.F. Chichibu, Appl. Phys. Express 13, 012004 (2020).

16. G. Oelgart, S. Gramlich, T. Bergunde, E. Richter, and M. Weyers, Mater. Sci. Eng. B44, 228 (1997).

17. K.-M. Song, D.-J. Kim, Y.-T. Moon, and S.-J. Park, J. Cryst. Growth 233, 439 (2001).

18. A. Saxler, D. Walker, P. Kung, X. Zhang, M. Razeghi, J. Solomon, W.C. Mitchel, and H.R. Vydyanath, Appl. Phys. Lett. 71, 3272 (1997).

19. P. Reddy, S. Washiyama, F. Kaess, R. Kirste, S. Mita, R. Collazo, and Z. Sitar, J. Appl. Phys. 122, 245702 (2017).

Publisher's Note Springer Nature remains neutral with regard to jurisdictional claims in published maps and institutional affiliations. 